\title{
Valuation, downside risk measures and asymmetric information: A portfolio optimization approach
}

\author{
Yoram Kroll ${ }^{1, ~ *, ~ M o s h e ~ B e n-H o r i n ~}{ }^{2}$ \\ ${ }^{1}$ Ono Academic College-Ono, Israel and Ruppin Academic Center- Hemek Hefer, Israel \\ ${ }^{2}$ Ono Academic College-Ono, Israel
}

\section{Email address:}

Kroll504@bezeqint.net (Y. Kroll), msmoney@ono.ac.il (M. Ben-Horin)

\section{To cite this article:}

Yoram Kroll, Moshe Ben-Horin. Valuation, Downside Risk Measures and Asymmetric Information: A Portfolio Optimization Approach. International Journal of Economics, Finance and Management Sciences. Vol. 2, No. 6, 2014, pp. 319-331. doi: $10.11648 /$ j.ijefm.20140206.14

\begin{abstract}
This paper proposed optimal equilibrium portfolio algorithm for valuing assets. When mean variance criterion is assumed, the proposed procedure and the conventional CAPM yield identical valuations. When a downside risk measures are employed and the distributions are asymmetric, the proposed algorithm and the three moments extensions of CAPM may yield close, but not necessarily identical, valuations. Our semi-variance results are identical to those of Bawa\& Lindenberg, but in contrast to those of Estrada's downside risk extension of CAPM. The impact on valuation of "Mean Variance Preserving Shifts" and asymmetrical information regarding future cash flows are demonstrated by the proposed model.
\end{abstract}

Keywords: Cash Flow Valuation, Semi-Variance, VaR, AVaR, DCF, Asymmetric Information, Stochastic Dominance

\section{Introduction}

The most common traditional approach to valuing financial assets and measuring an investment's impact on wealth (i.e., it's $N P V$ ), is to discount expected cash flows using the CAPM's Risk Adjusted Cost of Capital (RACC) discount rate. However, empirical studies showed that the traditional beta of CAPM provides very partial explanation for the variability of risky returns. Fama and French (1992, 1993, and 1995) seminal studies showed empirically that adding two additional factors (SMB and HML) improves dramatically the explanation of risky return. In this study we suggest a new algorithm for valuation that avoids many of the weak assumptions of the traditional CAPM. Empirical study may prove whether valuation according to the newly proposed algorithm with or without the SMB and HML factors of Fama and French improves the prediction of risky yields.

For more than fifty years ago studies have criticized the CAPM and its basic assumption that only the first two central moments of the distribution determine the optimal decision under uncertainty. Some have amended and extended the CAPM while others developed alternative approaches 1 .

1 Several authors have extended the CAPM and consider co-skewness and later also co-kurtusis. See Arditti (1967), Kraus and Litzenberger (1976), Friend and
Hanoch and Levy (1969), Rothschild and Stieglitz (1970), and Hadar and Russell (1971) developed the Stochastic Dominance (SD) efficiency criteria, which are based on all moments of the distribution. However, although the SD criteria are optimal for expected utility investors, they do not provide an algorithm to construct an optimal portfolio out of the given alternatives 2 .

It is well known that there is no unique measure of risk for all risk-averse utilities. Given two investors, $U$ and $V$, and two risky alternatives, $\mathrm{A}$ and $\mathrm{B}$, investor $\mathrm{U}$ may consider $\mathrm{A}$ riskier than $\mathrm{B}$ and agrees to pay a higher risk premium to avoid A's risk than he would to avoid B's risk. The opposite may hold for investor $\mathrm{V}$. Thus, there is no unique risk measure that can rank the risks of $\mathrm{A}$ and $\mathrm{B}$ for both $\mathrm{U}$ and $\mathrm{V}$. Nevertheless, we can reasonably search for a plausible risk measure that better approximates decision-making under risk. It is reasonable to claim that risk is related to the downside of the distribution of returns or cash flows and that risk is therefore associated with the probability of an investor obtaining a return or cash flow below a given reference

Westerfield (1980), Harvey and Siddique (2000), Dittmar (2002), Barone, Gagliardini and Urga (2004), Javid (2009) and others.

2 Levy and Markowitz (1979) demonstrated empirically that the expected utility of investors, who construct optimal mean variance portfolio, approximates very well the expected utility of investors who consider all moments of the distributions. 
point3. Harry Markowitz, the Nobel Prize laureate, declared in his 1990 Nobel lecture:

"Perhaps some other measure of portfolio risk will serve in a two parameter analysis for some of the utility functions which are a problem to variance. For example, in chapter 9 of Markowitz (1959), I proposed the 'semi-variance' $\mathrm{S}$ as a measure of risk where: $S=E\left(\operatorname{Min}(0, R-c)^{2}\right)$, where $c=E(R)$ or $c$ is a constant independent of choice of portfolio. Semivariance seems to be more plausible than variance as a measure of risk, since it is connected only with adverse deviations". Markowitz (1990), p. 286.

Baumol (1963) also recognized the deficiencies of variance as a risk measure for alternatives with different means. Baumol proposed "The Expected Gain-Confidence Limit Criterion", which is equal to the mean, $E$, minus $K$ standard deviations. Following these early works, many other studies have examined and supported the use of downside risk measure. 4

The present study examines valuation under equilibrium, where the variance is replaced by downside risk measures such as the semi-variance, VaR and AVaR. We also analyze the cases of symmetric and asymmetric information while assuming that the investors maximize their expected utility by maximizing the slope of their portfolios' tradeoff line between the expected rate of return and risk.

The proposed algorithm can be either employed as an alternative valuation approach to the traditional DCF approach or as an approach for calculating downside risk adjusted beta factor. In this study the optimal portfolio determines directly the value of the firm under equilibrium conditions while assuming that investors maximize the slope of the expected return-risk tradeoff line. Only when risk is measured by variance, then the DCF approach and our optimal portfolio valuation produce identical valuations. However, when we use a downside risk measure under nonnormal distribution conditions, then the equilibrium values under our approach differ from the value obtained by the conventional DCF approach and some of its extensions5. It should be noted that the valuation under the three moments extension of CAPM is not, and should not be, identical to the valuation by the below-mean variance algorithm presented here, despite some commonalities. The valuation according to Estrada's (2007) downside semi-variance beta has many conflicts with our semi-variance optimal portfolio valuation because there is no mathematical relationship between the co-skewness of the individual security and the skewness of the portfolio (See Cheremushkin (2009)).

Magni (2007, 2009) correctly claimed that using the RACC to calculate the value of a future cash flow involves a

3 The reference point can be, for example, the expected return, the risk free return, zero return or any other selected reference point.

4See reviews by Nawrocki (1999) and Post and Vilet (2006) and Ang, Chen and Xing. (2006).

5 For higher moments extensions of CAPM, see Harvey and Siddique (2000), and Kraus and Litzenberger (1976). For downside risk beta, see Bawa and Lindenberg (1977) and Estrada (2007). loop because the "correct" RACC should be computed only when the equilibrium value is known and employed in the calculation of RACC. Namely, the use of RACC for calculating values involves a loop since the correct equilibrium value is a required input for calculating the correct RACC. (See: Magni (2009)). In our paper we avoid this simultaneous loop since the value of an asset is determined directly from the optimal portfolio conditions without using the cost of capital.

\section{Preliminaries}

Bawa and Lindenberg (1977) define downside beta $\beta$ by:

$$
\beta^{-}=\frac{\operatorname{cov}\left(r_{i}, r_{m} \mid r_{m}<\mu_{m}\right)}{\operatorname{var}\left(r_{m} \mid r_{m}<\mu_{m}\right)}
$$

Where $r_{i}$ and $r_{m}$ are the security and market returns respectively and $\mu_{m}$ is the market expected return. Chen, Hang and Xing (2006) found a significant impact of $\beta$ - on the returns of securities. In case we select the risk measure to be the below mean semi-variance, then our valuation model yields identical results to the valuation of Bawa and Lindenberg. Estrada (2007) defined a downside beta $\beta^{D}{ }_{i}$ as:

$$
\boldsymbol{\beta}_{i}^{D}=\frac{E\left\{\min \left[\left(r_{i}-E\left(r_{i}\right), 0\right] \min \left[\left(r_{M}-E\left(r_{M}\right)\right), 0\right]\right\}\right.}{E\left[\min \left(r_{M}-E\left(r_{M}\right)\right), 0\right]^{2}}
$$

The single index CAPM of Estrada is then:

$$
E\left(r_{i}\right)=r_{F}+\beta_{i}^{D}\left[E\left(r_{M}\right)-r_{F}\right]
$$

Estrada (2007) presented empirical results and claims that a CAPM with downside beta better explains the behavior of risk and returns in the market. However, without disputing Estrada's empirical findings, we should note that Estrada's CAPM with a downside beta suffers from a serious deficiency because there is no mathematical relationship between $\beta_{i}^{D}$ of the individual securities and $\beta^{D}$ of the overall portfolio. Specifically, $\beta_{i}^{D}$ ignores hedging between the above mean returns and below mean returns. Our downside optimal portfolio valuation overcomes this deficiency because the valuation is based on the individual security's effect on the downside risk of the overall portfolio rather than on the downside risk of the individual security itself, which neglects hedging between the upside and downside returns.

Downside risk can be considered in many ways. This paper will apply in a numerical example the Value at Risk (VaR) and the Accumulated Value at Risk (AVaR).

Co-skewness is also related to downside risk and can also effect valuation. Namely, negative co-skewness generates higher downside risk and lower valuation. The impact of coskewness on CAPM was first developed by Kraus and Litzenberger (1976), who extended the conventional CAPM by adding $\beta$ for co-skewness:

$$
\beta(K L)_{S i}=\frac{E\left[\left(r_{i}-E\left(r_{i}\right)\right)\left(r_{m}-E\left(r_{m}\right)\right)^{2}\right]}{\mu_{3}\left(r_{m}\right)}
$$


Where $\mu_{3}\left(r_{m}\right)$ denotes the third central moment of the market's rate of return distribution in third root terms. This definition of the beta for co-skewness has discontinuity at zero skewness of the market portfolio, $\mu_{3}\left(r_{m}\right)$. When $\mu_{3}\left(r_{m}\right)$ is close to zero, (1) tends either to $+\infty$ or $-\infty$. When $\mu_{3}\left(r_{m}\right)<0$, a higher and positive numerator of (1) generates a lower and negative beta for co-skewness; when $\mu_{3}\left(r_{m}\right)>0$, a higher and positive numerator in (4) leads to the opposite results of a higher and positive beta for co-skewness. Harvey and Siddique (2000) solved this problem by redefining beta for co-skewness as:

$$
\beta(H S)_{S i}=\frac{\mathrm{E}\left(\left(r_{i}-E\left(r_{i}\right)\right)\left(r_{m}-E\left(r_{m}\right)\right)^{2}\right)}{\sigma\left(r_{i}\right) \sigma^{2}\left(r_{m}\right)}
$$

Note that the beta for co-skewness, when the security is the market portfolio, is 1 according to the two definitions of beta for co-skewness in (4) and (5).

Let $r_{i t}$ and $r_{m t}$ be the rates of return on the security and the market portfolio, respectively, in period $t$. We can estimate $\beta_{i}$ and $\beta(K L)_{S i}$ or $\beta(H S)_{S i}$ through a first pass time series regression:

$$
r_{i t}=\lambda_{i}+\beta_{i} r_{m t} \pm \beta_{s i} r_{m t}^{2}+\varepsilon_{i t}
$$

where the \pm sign is opposite to the skewness of the market portfolio when Kraus and Litzenberger's $\beta(K L)_{S i}$ is employed and negative when Harvey and Siddique's $\beta(H S)_{S i}$ is employed.

A second pass cross-section regression can be used to examine the explanatory power of the extended CAPM. Harvey and Siddique (2000) showed that the extended CAPM for co-skewness provides significant explanation for the returns, even when Fama and French's size and book-tomarket factors are considered.

The estimated cost of capital based on the extended CAPM model that includes co-skewness is6:

$$
\mathrm{k}_{\mathrm{e}}=\mathrm{E}\left(\mathrm{r}_{\mathrm{i}}\right)=\mathrm{r}_{\mathrm{F}}+\beta_{\mathrm{i}}\left[\mathrm{E}\left(\mathrm{r}_{\mathrm{m}}\right)-\mathrm{r}_{\mathrm{F}}\right] \pm \beta_{\mathrm{si}} \mathrm{E}\left(\mathrm{r}_{\mathrm{m}}^{2}\right)
$$

Where $r_{F}$ is the risk free rate, and the \pm sign is determined as explained above. The co-skewness and downside beta are two different ways, to extend the CAPM to account for asymmetry. These extensions can be applied to estimate the discounting factor in the process of valuation.

The Stochastic Dominance (SD) approach is a second way to overcome the deficiencies of the mean variance valuation approach. The SD efficiency analysis is based on all the moments of the distribution function.

Also our proposed algorithm is based on the actual distribution that reflects all moments of the distribution. Some parts the numerical example in this paper will consider the impact of shifts that are not reflected in mean and variance but generate SD preference of one distribution over

6 Harvey and Siddique (2002) defined the expected return on asset $i$ in period $t+1$ conditional on the estimates of $\beta_{i t}, s_{i t}$ and $k_{i t}$ and $E\left(r_{M t+1}\right), E\left(r_{M t+1}^{2}\right)$ and $E\left(r_{M t+1}^{3}\right)$. another. In the Appendix of this paper provides a short review of the SD approach and add some simple Theorems that will be used in some parts of the numerical example.

\section{The Model and the Optimization Algorithm}

Below, we present an algorithm for a simple "base case" equilibrium valuation7. We assume that the market consists of a very large number of firms, and each firm is very small (theoretically infinitesimally small) relative to the market. Namely, the cash flow of the individual firm (individual investment) is very small relatively to the aggregate cash flow of all the firms in the market. Thus the relative supply of cash flow of the individual investment is infinitesimally small. However, the size of each individual investment (firm) relative to other individual firms could be large and even very large, depending on the relative size of the anticipated cash flow and its characteristics. The demand for the cash flow of the individual investment is determined by the optimal portfolio considerations of the investors. If all investors have the same expectations, then equilibrium is obtained when the optimal holding of the individual investment is adequately very small. The degree of accuracy is determined by a small epsilon value set at the outset of the optimization algorithm. Given a very small epsilon value, the optimal proportion of each individual investment in the "market portfolio" is very small when the market is in equilibrium. One should bear in mind that though the proportion of each firm is by a small epsilon close to zero, the proportion of one stock can be very different the proportion of the other one. Under equilibrium the reward to risk of all stocks should be equal but the very small promotions of the individual stocks in the market can be relatively very different due to the very different size of the cash flows and the risk properties of the stocks' cash flow. Maximum expected utility is obtained by an investor when the Reward-to-Risk (RTR) of his portfolio is maximized8. The RTR is measured by the slope of a tradeoff line in the expected return-risk space that starts at the risk free rate and passes through the expected return and risk of the portfolio that contains the market portfolio and the individual security. It is assumed that the riskless rate of return and the bivariate distribution of market return and future cash flow of the individual investment are given. Thus, for any given present value of the investment the market's RTR is also known. In order to find the equilibrium present value of the individual investment we apply the following simple algorithm.

1. The algorithm begins by assuming an arbitrary market value for the individual investment. Given the end-ofperiod cash flow distribution and the investment's assumed value, we derive the investment's rate of return distribution. Given the bivariate distribution of the rate

\footnotetext{
7 Later, we present variations on the "base case".

8 Different measures of risk are simply different ways to better approximate expected utility in terms of expected return and risk
} 
of return on the investment and the rate of return on the market portfolio, the algorithm searches for the optimal proportion of the market portfolio and the individual investment that maximizes the RTR of the overall portfolio.

2. The lower the investment's initially assumed market value of the individual investment (in the first iteration of the algorithm), the higher its (initial) rate of return and the higher its (initial) proportion in the overall risky portfolio. This result occurs because, as noted above, we assume that the distribution of the investment's future cash flow is given. When the initial arbitrary value is set lower (higher) than the equilibrium value, the stock's marginal contribution of the individual investment to the market RTR is positive (negative), the demand for the stock drives its value up (down) and decreases (increases) its expected rate of return. This sets the stage for a decrease (increase) of the investment proportion in the stock in the ensuing iterations of the algorithm.

3. The process continues until the stock's marginal contribution to the overall portfolio's RTR is infinitesimally close to zero. Since the cash flow of the individual firm is assumed adequately small relative to the aggregate cash flows of all firms, this occurs when the optimal proportion of the stock of the individual firm in the overall portfolio of risky assets is adequately close to zero.

The assumption of a close to zero small epsilon raises immediately the question "how much small is small". For example Apple and Exxon are the biggest companies. Exxon, for example, makes up about $0.001(0.1 \%)$ of the US equity market's capital and much less than $0.1 \%$ of the world equity capital market. Our numerical example demonstrates that a stock's optimal investment proportion can be very sensitive to its equilibrium value. Later in our example, we show when we assume a semi-variance valuation of 113.831361, the optimal proportion (epsilon) approaches zero (0.000001\%) (See: the round number 113.83 in Table 3 below). However, when we lower the assumed value by $0.01 \%$ to 113.8199779 , then the optimal proportion rises to $0.303 \%$, which is three times higher than Exxon's proportion in the US equity market. If, in our example, we lower the value of 113.83 by $0.1 \%$, than the optimal value increases to almost $3 \%$ of the market. If the value decreases by $1 \%$, the optimal proportion increases to almost $20 \%$ of the market 9 . Clearly, the above examples of deviations from the close-to-zero valuation do not necessarily reflect the impact of such deviations in an empirical study. Namely, the empirical impact of the size of "epsilon" deviation from zero should be examined empirically. Also note that in case of asymmetrical information the more optimistic investor with respect to the future cash flows of the firm will hold above zero proportion of his wealth in the firm.

Let us now present in details some more specific assumptions behind our basic model.

9 Clearly, the above examples of deviations from the close-to-zero valuation do not necessarily reflect the impact of such deviations in an empirical study.
There are three types of securities in a one period, no tax, no trading costs, and a competitive market portfolio model10: A riskless asset that yields a rate of return $r_{f}$, which is the same for both borrowers and lenders, a market risky portfolio and the stock of a specific firm (investment). The firm's random cash flow is available only at the end of a single period. The multivariate distribution of this future cash flow and the random return of the market portfolio are given. . In the parts of this paper that examine valuation under asymmetric information, there are two types of investors with different views on the future bivariate distribution. The first type is the better-informed entrepreneur who, for example, initiates the firm at time zero and floats it in the market at the highest possible value. The second type of player is the lessinformed investor. Both types maximize their expected utility by controlling two decision variables: 1) the optimal investment proportion in the riskless asset and the complementary investment proportion in the risky portfolio; and 2) the proportion of investment in the equity of the specific firms and the risky (market) portfolio. In some parts of the analysis it is assumed that the less-informed investors might undervalue the investment. In that case, the entrepreneur's optimal proportion in the investment is positive and does not approach zero, and the total amount that he invests in the firm depends on his wealth and his optimal investment in the risky portfolio.

A key element in the expected utility maximization approach is the assumption that expected utility is approximated by a function of expected return and risk, and then the immediate question is related to the appropriate measure of risk. As mentioned before, in general there is no unique measure of risk that suits all expected utility investors. However, it is reasonable to assume that in cases of non-normal distributions, downside risk measures are more appropriate than variance as well as other two-sided spread measures.

The Taylor Expansion of the expected utility around the portfolio mean $\left(\mu_{1}\right)$ is:

$$
\begin{aligned}
\mathrm{EU}(\mathrm{)}= & \mathrm{U}\left(\mu_{1}\right)+\mathrm{U}_{2}\left(\mu_{1}\right) \cdot \mu_{2} / 2 !+\mathrm{U}_{3}\left(\mu_{1}\right) \cdot \mu_{3} / 3 !+\mathrm{U}_{4}\left(\mu_{1}\right) . \\
& \mu_{4} / 4 !+\ldots+\mathrm{U}_{\mathrm{N}}\left(\mu_{1}\right) \cdot \mu_{\mathrm{N}} / \mathrm{N} !+\mathrm{R}_{\mathrm{N}+1}
\end{aligned}
$$

Where $U_{i}$ is the $i^{t h}$ derivative of the utility function; $\mu_{i}$ is the $i^{\text {th }}$ central moment of the distribution of the returns; and $R_{N+1}$ is the $N+1$ residual. It is clear from (8) that, generally, all moments of the distribution affect the investor's expected utility. We can approximate the expected utility as a function of expected return and risk as follows:

$$
\text { EU ( ) }=f\left(\mu_{1}, \operatorname{Risk}\left(\mu_{1}, \mu_{2}, \mu_{3}, \mu_{4}, \ldots\right)\right) \text {. }
$$

In the case of normal distributions, all moments higher than $\mu_{2}$ are either zero or functions of $\mu_{1}$ and $\mu_{2}$. Thus, we can express the expected utility as:

$$
E U()=f\left(\mu_{1}, \operatorname{Risk}\left(\mu_{1}, \mu_{2}\right)\right)
$$

10 To extend the analysis of this paper to the multiperiod case, one can use a recursive technique in which the value of an asset at $N_{t-1}$ is calculated by the random cash flows of the asset at time $N_{t}$. Alternatively, one can extract a discounting rate out of the one period optimal portfolio valuation and use this discounting rate in the multiperiod scenario. 
In the normal distribution case, the mean and variance of the distribution fully characterize the distribution, and, thus, the $(M-V)$ efficiency rule is a perfect selection and ranking rule for all investors. However, when the return distribution in not normal, asymmetry, kurtosis $\left(\mu_{3}\right.$ and $\left.\mu_{4}\right)$ and higher moments may affect the investor's preferences and the $M-V$ rule may yields a poor approximation of the expected utility ranking compared to the ranking of mean-downside risk measures 11.

The conventional CAPM assumes that maximizing expected utility is equivalent to maximizing the well-known Sharpe's Reward-to-Variability ratio $(R V)$ :

$$
R V=\frac{\left(E(R)-R_{f}\right)}{V^{0.5}}
$$

In addition to the standard deviation, risk measure in the denominator of (11), this paper applies the below-mean semivariance (SV), VaR and AVaR for given confidence level (1p). The resulting expected reward to risk (RTR) measures are:

The expected Reward-to-Semi-Variance $(R S V)$ is 12 :

$$
R S V=\frac{\left(E(R)-R_{f}\right)}{S V^{0.5}}
$$

Expected reward to $\mathrm{VaR}$

$$
\operatorname{RVaR}(\mathrm{p})=\frac{\left(E(R)-R_{f}\right)}{\operatorname{VaR}(p)}
$$

And expected reward to AVaR

$$
\operatorname{RAVaR}(\mathrm{p})=\frac{\left(E(R)-R_{f}\right)}{A \operatorname{VaR}(p)}
$$

Similarly to Sharpe's ratio, $\operatorname{RVaR}(\mathrm{p})$ and $\operatorname{RAVaR}(\mathrm{p})$ are the slopes of a straight tradeoff lines between expected return and risk.

The next section numerically compares the proposed downside risk measure valuations with the valuations of four alternative models: the conventional CAPM, the Kraus and Litzenberger (1976) model, the Harvey and Siddique (2000) model, Bawa and Lindenberg (1977) and Estrada's (2007) model. Under asymmetric information, when, for example, the less informed market investors undervalue the investment, then only this study's valuation algorithm can determine the proportion of the investment that the entrepreneur would retain in his optimal risky portfolio. It will be shown that in the case of asymmetric information, MVPS shifts can have a significant effect on the entrepreneur's decisions.

\section{Numerical Examples}

Here, we present three examples that differ in the joint distribution of cash flows and market returns. Each example

11 See Danielson, Jorgensen, Serma and Vries (2005).

12 It is also possible to define risk as semi-variance below the risk free rate, below zero or below any other reference rate. consists of two cases. The first is the symmetric information case, and the second demonstrates the asymmetric information case13. Each example is presented with a table that consists of four panels. Panel A presents the bivariate distribution of the cash flows and the market returns. Panel B presents some basic statistics. Panel $\mathrm{C}$ provides valuations according to the CAPM, the four alternative extensions of CAPM models and the two versions of the proposed model of this paper. Panel D, which relates to the asymmetric information case, presents the maximum percentage of the firm that the better-informed entrepreneur would agree to sell for any given market valuation.

\section{Example 1: Symmetric Market Returns and Symmetric Cash Flows}

The Symmetric Information Case. Panel A of Table 1 presents a highly simplified example where the joint distribution of the cash flow of a particular firm, Firm A, and the market return is a discrete and bivariate uniform distribution14. There is thus no skewness and no correlation between the cash flows and the market rates of return.

Table 1. Valuation with Bivariate Distribution of Returns: Zero Skewness and Zero Correlation

Panel 1-A. The Bivariate Distribution of the Cash Flows and the Market Returns

\begin{tabular}{lllllll}
\hline \multirow{2}{*}{$\begin{array}{l}\text { Investment's } \\
\text { Cash Flows }\end{array}$} & \multicolumn{6}{l}{ Market Rates } \\
\cline { 2 - 7 } & $\mathbf{- 5 0}$ & $\mathbf{- 2 0 \%}$ & $\mathbf{1 0 \%}$ & $\mathbf{4 0 \%}$ & $\mathbf{7 0 \%}$ & $\begin{array}{l}\text { Marginal } \\
\text { Probability }\end{array}$ \\
\hline 60 & $5 \%$ & $5 \%$ & $5 \%$ & $5 \%$ & $5 \%$ & $25 \%$ \\
100 & $5 \%$ & $5 \%$ & $5 \%$ & $5 \%$ & $5 \%$ & $25 \%$ \\
140 & $5 \%$ & $5 \%$ & $5 \%$ & $5 \%$ & $5 \%$ & $25 \%$ \\
180 & $5 \%$ & $5 \%$ & $5 \%$ & $5 \%$ & $5 \%$ & $25 \%$ \\
Marginal & $20 \%$ & $20 \%$ & $20 \%$ & $20 \%$ & $20 \%$ & $100 \%$ \\
Probability & & & & & & \\
\hline
\end{tabular}

Panel 1- B. Statistics

\begin{tabular}{lll}
\hline & Cash Flow & Market Returns \\
\hline Mean & 120.00 & $10.00 \%$ \\
Standard deviation & 44.72 & $42.43 \%$ \\
Skewness $^{2}$ & 0.00 & $0.00 \%$ \\
Kurtosis $^{2}$ & 50.61 & $48.44 \%$ \\
VaR(10\%),VaR(20\%) & 40,40 & $50 \%, 50 \%$ \\
AVAR(10\%),AVAR $(20 \%)^{1}$ & 40,40 & $50 \%, 50 \%$ \\
Correlation & 0.00 & \\
Co-Skewness HS and KL & 0.0 And not defined (division by zero), \\
\hline
\end{tabular}

${ }^{1}$ VaR and AVaR are below zero for amounts of the cash flow and percentage for Market's returns.

${ }^{2}$ The skewness is in third root terms, and the kurtosis is in fourth root terms.

13 Note, that the numerical example is intended only to examine the basic features of the proposed algorithm. An empirical test related to returns and downside risk may provide a more rigorous examination of the model.

14 To analyze the data and apply the algorithm, a bivariate discrete uniform distribution presented in a $5^{\times} 4$ matrix is used. A spreadsheet with the distribution and all the relevant calculations are available from the author. 
Panel 1-C. Valuation under Equilibrium and Symmetric Information

\begin{tabular}{llll}
\hline $\begin{array}{l}\text { Valuation Method/ Risk } \\
\text { Measure }\end{array}$ & Beta & RACC & Value \\
\hline Traditional CAPM & 0 & $4 \%$ & 115.38 \\
Kraus \& Litzenberger & 0 & $4 \%$ & 115.38 \\
Harvey \& Siddique & 0 & $4 \%$ & 115.38 \\
Bawa \& Lindenberg & 0 & $4 \%$ & 115.38 \\
Estrada & 0.357 & $6.12 \%$ & 113.08 \\
Variance & & $4 \%$ & 115.38 \\
Semi-Variance & & $4 \%$ & 115.38 \\
VaR(10\%) & & & $111.98-119.73$ \\
Var(20\%) & & & $115.24-115.77$ \\
AVaR(10\%) & & & $111.98-117.94$ \\
AVaR(20\%) & & & $113.89-117.94$ \\
\hline
\end{tabular}

${ }^{1}$ Due to the discrete CDF, the discontinuity problem of the first and second derivatives was reduced by using third degree trend line polynomial approximation of the DCF. Even with this approximation the equilibrium Valuation can be in arrange rather than in a single valuation point. The higher is the confidence level (1-p) the higher is the confidence level (1-p) the lower is t range of VaR.

Panel 1-D. The Entrepreneur's Maximum Proportion of Wealth, Invested in the Firm for Given Values of the Firm and Current Paper Risk Measure

\begin{tabular}{llllll}
\hline Firm's Value & 115 & 100 & 90 & 80 & 70 \\
variance & $6.5 \%$ & $70.6 \%$ & $78.1 \%$ & $81.5 \%$ & $83.2 \%$ \\
semi-variance & $6.5 \%$ & $70.6 \%$ & $78.1 \%$ & $81.5 \%$ & $83.2 \%$ \\
\hline
\end{tabular}

The traditional CAPM and its three moments' extensions and Bawa and Lindenberg downside extensions calculate zero betas for risk and for skewness (Panel 1-C). The expected future cash flow of 120 is thus discounted by the assumed risk-free rate of $4 \%$ to obtain a present market value of 115.38 (Panel 1- C). Our algorithm obtained the same valuation, when Sharpe's $R V$ ratio or Reward-to-SemiVariance $(R S V)$ ratio are used. The results of Estrada valuation are very different from all the others including those of Bawa and Lindenberg (1977) that considered downside beta. Valuation according to Estrada is only 113.08 instead of 115.38 and the discounting rate is $6.12 \%$ rather than $4 \%$. In other words, there is no mathematical relationship between the Estrada's below mean distribution of returns of the individual securities and that of the portfolio (see Cheremushkin (2009)). Our model's $R S V$ does not suffer from this deficiency because $R S V$ is defined in terms of the overall portfolio, and not the individual securities.

According to our algorithm, valuation using $\mathrm{VaR}$ and $\mathrm{AVaR}$ risk measures is the based on data from the cumulative distribution function (CDF). Unfortunately, our simplified numerical examples contain only five market returns and four cash flows of the firm. This limited data is used to construct the discrete CDF of the risky portfolio. Due to the discrete $\mathrm{CDF}$, the reward to VaR function has discontinuity points that disturb the optimal portfolio algorithm. To avoid this problem we replace the discrete $\mathrm{CDF}$ by a third degree polynomial trend line approximation for the CDF. This approximation enables us to apply the approximated VaR risk measure and to find a range for available equilibrium values. For Example in case the VaR $(10 \%)$ risk measure is applied, the obtained range is 111.98-119.73 (See Panel 1-c). This wide range of values happens due to the discontinuity problem of the second derivative of the Maximum reward to VaR function when the proportion of the market portfolio approaches $100 \%$ and the proportion of the firm in the risky portfolio approaches zero (see also Figure 1). However, when we replace the VaR $(10 \%)$ by VaR $(20 \%)$ the range of equilibrium value is reduced dramatically to $115.24-115.77$. This range is a narrow range around the 115.38 value obtained by the other six methods. We do believe that large number of empirical distribution points should minimize the range of equilibrium value when VaR risk measure is considered. When AVaR risk measure is applied, a range of equilibrium value is obtained without the need to apply trend line approximation. Once again due to discontinuity of the second derivative, obtain a range of valuation. The range for AVaR (10\%) of $111.98-117.94$ is wider than the range for AVaR (20\%) of 113.89-117.94. This result supports our belief that in a more realistic case that will be based on a large sample of empirical data the range will be much narrower.

The Asymmetric Information Case. Here, we consider a case where one investor (the "entrepreneur") is betterinformed about the firm than the rest of the investors, who are "less-informed". The entrepreneur values the cash flow correctly, and the other investors undervalue the investment. Out of all the models presented here, only our optimal portfolio model can consider this case. Panel 1-D presents, for alternative market valuations, the maximum investment proportion of the risky portfolio that the entrepreneur will allocate to the firm when variance and semi-variance risk measures are employed. For example, if the equilibrium market value of firm is undervalued to 115 (rather than 115.38), then the entrepreneur allocates up to $6.5 \%$ of his risky portfolio to the firm. If the market undervalues the firm to 100 , the entrepreneur will allocate up to $70.6 \%$ of his risky portfolio in the firm. The maximum optimal proportion in the risky portfolio that is allocated by the entrepreneur to the firm when VaR and AVaR risk measures are employed can be also observed in Figure 1.

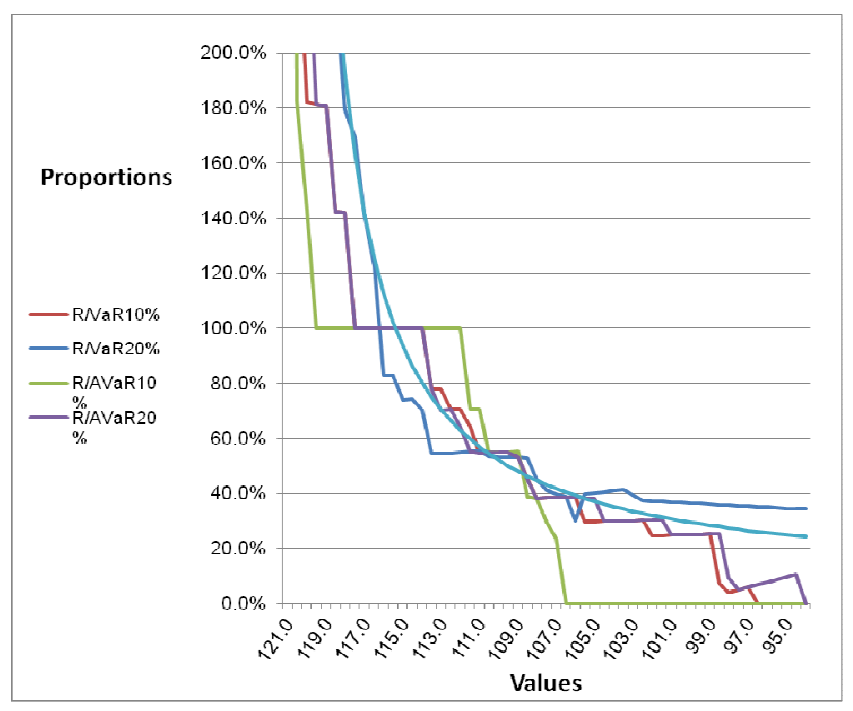

Figure 1. Optimal proportions of market portfolio for given values of the firm, Based on data in Table 1 
This Figure shows that when variance is the risk measure and reward to standard deviation (R/STDV) is maximized by investors, then the optimal proportion of the market portfolio that will be held by the entrepreneur is a downward convex function of the assumed value of the firm. However, when the VaR or AVaR risk measured are employed, the optimal proportion of the market tends to decrease when the value of the firm decreases. However, it does not decrease monotonically and especially there is a wide range of valuations that fits optimal proportion of $100 \%$. It is reasonable to assume that when DCF will include more points, the non-monotony, the non-convexity problem will be minimized.

Let formalize the asymmetrical information case. Let $W_{0}$ be the initial wealth of the better-informed investor (i.e., the entrepreneur). Denote the optimal proportion of wealth invested by the entrepreneur in his risky portfolio by $p$ (and the proportion $q=1-p$ is invested in the riskless asset). In addition, let $p^{\mathrm{F}}$ denotes the entrepreneur's optimal proportion in firm F (of which he is "better-informed") out of his portfolio of risky assets. Let $V_{0}$ denote the equilibrium market value of the firm $\mathrm{F}$ (as determined in the market, in which the less-informed investors and the entrepreneur compete for firm's stock). Then, $I$, the total amount of capital invested by the entrepreneur in firm $\mathrm{F}$ is:

$$
I=\operatorname{Min}\left[p^{F} p W 0, V_{0}\right]
$$

If the entrepreneur is wealthy (or if the investment is small enough) and $p^{F} p W_{0}>V_{0}$, then the entrepreneur will retain all of the firm's equity. Otherwise, he will hold $p^{F} p W_{0}$ of the equity. In Case $\mathrm{D}$ of Table 1 , when $V_{0}=100$, the amount invested by the entrepreneur in the firm is $I=\operatorname{Min}\left[70.6 \% \mathrm{p} W_{0}\right.$, 100]. The entrepreneur will allocate $70.6 \%$ of his risky portfolio to the firm if $0.706 p W_{0}<100$. Otherwise, he will retain all of the firm's equity, and $p^{F}$ will be less than 0.706 (and equal to $100 / p W_{0}$ ). Note that the optimal $p$ is subjective and depends on the investor's wealth and utility function and the firm's undervaluation in the market. A larger market undervaluation makes the investment more attractive to the better-informed entrepreneur, and he allocates more wealth to the firm15. If, for example, the undervaluation is extensive and $V_{0}=90$, then the entrepreneur's maximum investment proportion in the firm is $78.1 \%$ of his risky portfolio (See Table 1 panel D)16. Note that, under asymmetric information, the market discount factor is irrelevant to the entrepreneur who diversifies his investment differently than the uninformed investors that determine the value of the firm in the market.

\section{Example 2: Negatively Skewed Market Returns and Positively Skewed Cash Flows}

The Symmetric Information Case. Table 2 presents a

15 The optimization of this part is beyond the scope of this paper.

16 In this specific example, due to convexity at a value of 57.66 there is a minimum holding of $100 \%-16.39 \%$. In the other examples in the paper, the minimum is at a short position of the firm. discrete bivariate distribution of the investment cash flows and the market returns with a positive correlation of 0.269 between the firm's cash flows and the market portfolio's returns. The market return distribution exhibits a negative skewness of $-16.52 \%$. The cash flow has a positive skewness of 26.78, and a positive Harvey and Siddique's co-skewness measure of 0.0105 . All skewness measures are in third root terms. According to the traditional CAPM, the valuation is 113.75 (compared with 115.38 in Table 1) because the equilibrium beta for risk is 0.249 . The resulting RACC is $4 \%+1.50 \%=5.50 \%$. Our method obtains the same valuation when variance is the risk measure. However, when the portfolio's semi-variance replaces the variance, the equilibrium beta for risk is 0.237 . The resulting RACC is $4 \%+1.42 \%=5.42 \%$, and our algorithm obtains the value 113.83, which is slightly higher than the traditional CAPM's 113.75 value and lower than the comparable value calculated in Table 117. Exactly the same valuation is obtained by Bawa and Lindenberg's downside risk measure. The values that the three moments CAPM of Kraus and Litzenberger (113.84) and Harvey and Siddique (113.97) 18,19 obtained are also smaller than the 115.38 value presented in Table 1 . Once again, the valuation obtained by Estrada's semi-variance model is in the opposite direction; Estrada's value for the Table 2 data is 112.39, compared to the higher value of 113.08 for the Table 1 data. The positive co-skewness in Table 2 yields a higher valuation and, thus, lower discount rates. This effect is reflected in the three moments CAPM by adding a beta for the skewness of -0.0046 for KL and 0.0105 for HS's measure of co-skewness. According to our semi-variance model, the beta is 0.237 , and the value is 113.83 ; these results can be compared to the beta of 0.249 and value of 113.75. The semi-variance beta of 0.237 is composed of two parts: the conventional beta of 0.249 and a (negative) difference of -0.012 , which reflects the co-non-normality. Note that once again when VaR or AVaR risk measures are employed we obtain only a range of valuation. The range is narrower once for $20 \%$ rather than $10 \%$ VaR or AVaR are employed.

17 This assumes a one period framework. If these valuation differences persist over 25 periods, our initial value would be higher than the CAPM's value by $1.77 \%$, calculated as follows: $(113.83 / 113.75)^{\wedge} 25-1=1.77 \%$.

18 Note in Table 2 that beta for skewness of Kraus and Litzenberger is negative while the one according to Harvey and Siddique is positive. This is not a conflicting result. When the skewness of the market is negative, the co-skewness in the denominator of (6) should be multiplied by -1 since in case of negatively skewed market returns, investors dislike positive co-skewness.

19 Also note that valuation by Kraus and Litzenberger can be different from that of Harvey and Siddique as their beta measure for co-skewness is not the same. Also note that these two valuations should not be the same as the one proposed by this paper, since the risk measures can be different, and since semi-variance is affected only indirectly by skewness as well as by other moments. However, the direction of the valuation's results is the same under equilibrium with symmetric information according to these three methods. 
Table 2. Valuation with Bivariate Distribution of Returns: The Positive Skewness of Cash Flows, Negative Skewness of Market Returns and Positive Correlation

Panel 2- A. The Bivariate Distribution of the Cash Flows and the Market Returns

\begin{tabular}{lcccccl}
\hline \multirow{2}{*}{$\begin{array}{l}\text { Investment's } \\
\text { Cash Flows }\end{array}$} & $\mathbf{- 5 0 \%}$ & $\mathbf{- 2 0} \%$ & $\mathbf{1 2 \%}$ & $\mathbf{3 6 . 9 \%}$ & $\mathbf{7 1 . 9 \%}$ & $\begin{array}{l}\text { Marginal } \\
\text { Probability }\end{array}$ \\
\cline { 2 - 7 } & $7.5 \%$ & $3.0 \%$ & $4.0 \%$ & $5.0 \%$ & $0.5 \%$ & $20 \%$ \\
60 & $9.5 \%$ & $8.0 \%$ & $8.0 \%$ & $8.0 \%$ & $6.5 \%$ & $40 \%$ \\
100 & $1.5 \%$ & $0.0 \%$ & $2.0 \%$ & $4.0 \%$ & $2.5 \%$ & $10 \%$ \\
140 & $3.5 \%$ & $5.0 \%$ & $6.0 \%$ & $7.0 \%$ & $8.5 \%$ & $30 \%$ \\
180 & $22 \%$ & $16 \%$ & $20 \%$ & $24 \%$ & $18 \%$ & $100 \%$ \\
Marginal & & & & & & \\
Probability & &
\end{tabular}

Panel 2- B. Statistics

\begin{tabular}{lll}
\hline & Cash Flow & Market Returns \\
\hline Mean & 120.00 & $10.00 \%$ \\
Standard deviation & 44.72 & $42.43 \%$ \\
Skewness & & $-16.52 \%$ \\
VaR(10\%),VaR(20\%) & 40,40 & $50 \%, 50 \%$ \\
AVaR(10\%),AVaR(20\%) & 60,45 & $75 \%, 62.5 \%$ \\
Kurtosis & 50.61 & $48.96 \%$ \\
Correlation & 0.269 & \\
Co-Skewness & 0.010494 and -0.00464, respectively \\
\hline
\end{tabular}

Panel 2-C. Valuation under Equilibrium and Symmetric Information

\begin{tabular}{|c|c|c|c|c|c|c|}
\hline \multicolumn{2}{|c|}{$\begin{array}{l}\text { Valuation Method/ } \\
\text { Risk Measure }\end{array}$} & $\begin{array}{l}\text { Beta- } \\
\text { Risk }\end{array}$ & $\begin{array}{l}\text { Beta- } \\
\text { Skew }\end{array}$ & RACC & \multicolumn{2}{|c|}{ Value } \\
\hline \multicolumn{2}{|c|}{ Traditional CAPM } & 0.249 & --- & $5.50 \%$ & \multicolumn{2}{|c|}{113.75} \\
\hline \multicolumn{2}{|c|}{$\begin{array}{l}\text { Kraus \& } \\
\text { Litzenberger }\end{array}$} & 0.249 & -0.0046 & $5.41 \%$ & \multicolumn{2}{|c|}{113.84} \\
\hline \multicolumn{2}{|c|}{ Harvey \& Siddique } & 0.249 & 0.0105 & $5.29 \%$ & \multicolumn{2}{|c|}{113.97} \\
\hline \multicolumn{2}{|c|}{ Bawa \& Lindenberg } & 0.237 & & $5.42 \%$ & \multicolumn{2}{|c|}{113.83} \\
\hline \multicolumn{2}{|c|}{ Estrada } & 0.462 & --- & $6.77 \%$ & \multicolumn{2}{|c|}{112.39} \\
\hline \multicolumn{2}{|c|}{ Variance } & 0.249 & --- & $5.50 \%^{2}$ & \multicolumn{2}{|c|}{113.75} \\
\hline & 0.237 & --- & $5.42 \%^{2}$ & \multicolumn{2}{|c|}{113.83} \\
\hline \multicolumn{2}{|c|}{$\operatorname{VaR}(10 \%)^{1}$} & & & & \multicolumn{2}{|c|}{$112.75-117.73$} \\
\hline \multicolumn{2}{|c|}{$\operatorname{VaR}(20 \%)^{1}$} & & & & \multicolumn{2}{|c|}{$115.42-115.27$} \\
\hline \multicolumn{2}{|c|}{$\operatorname{AVaR}(10 \%)^{1}$} & & & & \multicolumn{2}{|c|}{$110.90-118.77$} \\
\hline \multicolumn{2}{|c|}{$\operatorname{AVaR}(20 \%)^{1}$} & & & & \multicolumn{2}{|c|}{$112.45-116.45$} \\
\hline \multicolumn{7}{|c|}{$\begin{array}{l}{ }^{1} \text { Due to the discrete CDF, the discontinuity problem of the first and second } \\
\text { derivative was reduced by using third degree trend line polynomial } \\
\text { approximation of the DCF. Even with this approximation the equilibrium } \\
\text { Valuation can be in arrange rather than in a single valuation point. The } \\
\text { higher is the confidence level (1-p) the higher is the confidence level (1-p) } \\
\text { the lower is t range of VaR. } \\
{ }^{2} \text { The implied cost of capital for a multiperiod analysis. }\end{array}$} \\
\hline \multicolumn{7}{|c|}{$\begin{array}{l}\text { Panel 2-D. The Entrepreneur's Maximum Proportion of Wealth, Invested in } \\
\text { the Firm for given Values of the Firm and the Paper Risk Measure }\end{array}$} \\
\hline \multirow{3}{*}{$\begin{array}{l}\text { Firm's } \\
\text { Value } \\
\text { variance } \\
\text { semi- } \\
\text { variance }\end{array}$} & 115 & 100 & 90 & 80 & & 70 \\
\hline & $-29.58 \%$ & $87.03 \%$ & 103.8 & 115. & & $126.71 \%$ \\
\hline & $-30.73 \%$ & $92.89 \%$ & 106.4 & 115. & & $124.46 \%$ \\
\hline
\end{tabular}

The Asymmetric Information Case. When there is a positive correlation between the cash flows of the firm and the market return (Table 2) under the condition of symmetric information, the "mean variance entrepreneur" will give up almost all his holdings in the cash flow of the investment when the price is 113.75 . In contrast, the "mean semivariance" investor will give up all his holdings at the somewhat higher price of 113.83. However, under asymmetric information and market undervaluation, this difference can be much more significant. For example, the price in the market is only 100 the "mean variance entrepreneur" holds in the firm up to $87.03 \%$ of his risky investment and the "mean semi-variance entrepreneur" that favors the positive co-skewnes holds in the firm up to $92.89 \%$.

\section{Example 3: Negatively Skewed Market Returns and Negatively Skewed Cash Flows}

The Symmetric Information Case. We obtained the cash flow distribution presented in Table 3 by adding a "Mean Variance Preserving Step" (MVPS) to the distribution data of Table 2. As a result, the cash flow's skewness was reduced, but the variance, co-variances and the co-skewness matrix remained unchanged. Whereas the third root of the cash flow skewness in Table 2 is 26.78 , the comparable skewness in Table 3 is -26.78 . As we expected for symmetric information and equilibrium, this MVPS did not change any of the valuations, including Estrada's. An increase in skewness without a change in co-skewness is equivalent to a change of an idiosyncratic risk.

Table 3. Valuation after Adding Mean-Variance Preserving Shifts (MVPS), Which Lower Skewness, to the Cash Flow of Table 2

Panel 3-A. The Bivariate Distribution of the Cash Flows and the Market Returns

\begin{tabular}{lcccccl}
\hline \multirow{2}{*}{$\begin{array}{l}\text { Investment's } \\
\text { Cash Flows }\end{array}$} & \multicolumn{2}{l}{ Market Rates } & & & & \\
\cline { 2 - 7 } & $\mathbf{5 0 \%}$ & $\mathbf{- 2 0 \%}$ & $\mathbf{1 2 \%}$ & $\mathbf{3 6 . 9 \%}$ & $\mathbf{7 1 . 9 \%}$ & $\begin{array}{l}\text { Marginal } \\
\text { Probability }\end{array}$ \\
\hline 60 & $9.5 \%$ & $5.0 \%$ & $6.0 \%$ & $7.0 \%$ & $2.5 \%$ & $30 \%$ \\
100 & $3.5 \%$ & $2.0 \%$ & $2.0 \%$ & $2.0 \%$ & $0.5 \%$ & $10 \%$ \\
140 & $7.5 \%$ & $6.0 \%$ & $8.0 \%$ & $10.0 \%$ & $8.5 \%$ & $40 \%$ \\
180 & $1.5 \%$ & $3.0 \%$ & $4.0 \%$ & $5.0 \%$ & $6.5 \%$ & $20 \%$ \\
Marginal & $22 \%$ & $16 \%$ & $20 \%$ & $24 \%$ & $18 \%$ & $100 \%$ \\
Probability & & & & & & \\
\hline
\end{tabular}

Panel 3-B. Statistics

\begin{tabular}{lll}
\hline & Cash Flow & Market Returns \\
\hline Mean & 120.00 & $10.00 \%$ \\
Standard deviation & 44.72 & $42.43 \%$ \\
Skewness $^{1}$ & -26.78 & $-16.52 \%$ \\
Kurtosis $^{1}$ & 50.61 & $48.96 \%$ \\
VaR10\%,VaR20\% & $40.00,40.00$ & $50.00 \%, 50.00 \%$ \\
AVaR10\%,AVaR20\% & $76.00,57.00$ & $95.00 \%, 71.25 \%$ \\
Correlation & 0.269 & \\
Co-Skewness & 0.010494 and -0.00464, respectively \\
\hline
\end{tabular}


Panel 3-C. Valuation under Equilibrium and Symmetric Information

\begin{tabular}{lllll}
\hline $\begin{array}{l}\text { Valuation Method/ } \\
\text { Risk Measure }\end{array}$ & $\begin{array}{l}\text { Beta- } \\
\text { Risk }\end{array}$ & $\begin{array}{l}\text { Beta- } \\
\text { Skew }\end{array}$ & RACC & Value \\
\hline Traditional CAPM & 0.249 & --- & $5.50 \%$ & 113.75 \\
Kraus \& Litzenberger & 0.249 & -0.0045 & $5.41 \%$ & 113.84 \\
Harvey \& Siddique & 0.249 & 0.0105 & $5.29 \%$ & 113.97 \\
Bawa \& Lindenberg & 0.237 & -- & 5.42 & 113.83 \\
Estrada & 0.462 & --- & $6.77 \%$ & 112.39 \\
Variance & 0.249 & --- & $5.50 \%{ }^{2}$ & 113.75 \\
Semi-variance & 0.237 & --- & $5.42 \%{ }^{2}$ & 113.83 \\
VaR(10\%) & & & & $112.76-117.75$ \\
${\text { VaR }(20 \%)^{1}}^{1}$ & & & & $115.19-115.24$ \\
AVaR $(10 \%)^{1}$ & & & & $110.50-118.40$ \\
AVaR $(20 \%)^{1}$ & & & & $112.34-116.20$ \\
\hline
\end{tabular}

${ }^{1}$ The skewness is in third root terms, and the kurtosis is in fourth root terms.

${ }^{2}$ The implied cost of capital for a multi-periods analysis.

Panel 3- D. The Entrepreneur's Maximum Proportion of Wealth, Invested in the Firm for Given Values of the Firm and Current Paper Risk Measure

\begin{tabular}{llllll}
\hline Firm's Value & 115 & 100 & 90 & 80 & 70 \\
variance & $-29.6 \%$ & $87.0 \%$ & $103.8 \%$ & $115.5 \%$ & $126.7 \%$ \\
Semi-variance & $-32.9 \%$ & $78.9 \%$ & $100.1 \%$ & 115.7 & $131.1 \%$ \\
\hline
\end{tabular}

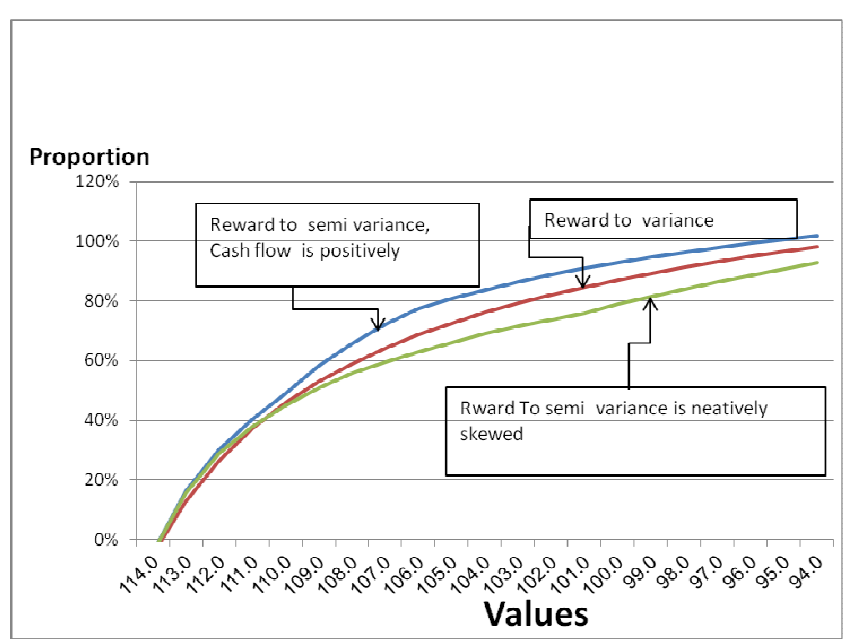

Figure 2. Optimal proportions of the firm as a function of its value and risk measures, based on data from Tables 2 and 3.

The Asymmetric Information Case. In both cases idiosyncratic change does not affect equilibrium value of the firm but do affect optimal investment of investors that over or under invest in the firm due to heterogeneous expectations. When we assume a "mean semi-variance entrepreneur" and asymmetric information, the valuation is affected. When the skewness of the cash flow is negative, as in Table 3 , and the value of the firm is only 100 , the "mean semi-variance entrepreneur" is willing to hold up to $78.9 \%$ of the firm where in case there is a positive skweness of the cash flow, as in Table 2, the entrepreneur is willing to hold up to $92.89 \%$ of the firm. Figure 2 presents the maximum optimal proportion invested in the firm by the better informed investor as a function of the market value of the firm.

According to the data of Tables 2 and 3, when there are no heterogeneous expectations, all type of investors invest in the firm a proportion that is close to zero when the value of the firm is 113.8. All types of better informed investor invest more in the firm when the firm is more undervalued. The mean variance better informed investor does not change his decision due to a change in idiosyncratic skewness. Namely, his optimal proportion of investment in the firm is the same when the firm has positive skewness (The case in Table 2) or negative one (The case in Table 3). However, the semivariance better informed investors change their optimal proportion due to a change in skewness. In case of Table 2 when the cash flow has positive skewness the semi variance investor invest more than the variance investor when the firm is undervalued. The opposite holds in case of Table 3 when the firm has negative skewness (See Figure 2).

\section{Conclusion}

This paper proposes an optimal portfolio valuation algorithm that assumes any selected risk measures (downside risk measures such as semi-variance or value at risk or any other selected risk measure). Empirical examination of the proposed algorithm can determine whether the proposed algorithm adds explanation to return above the traditional CAPM approach as well as its Fama \&French and the higher moments extensions. This study uses optimization that is based on maximizing the overall portfolio's Reward-to-Risk (RTR), and the algorithm is robust to definitions of risk. Thus, it is suitable for a variety of risk definitions. When variance is the risk measure, then the algorithm's valuation is identical to that of the conventional CAPM. The proposed algorithm yields the same results to those of Bawa and Lindenberg (1977) when semi variance is our risk measure. Our results are close but not identical to valuations by the three moments' extensions of CAPM (Krause and Litzenberger, (1976); Harvey and Siddique, (2000)). The semi variance valuation that is presented in this paper is not consistent with Estrada's (2000) valuation because there is no mathematical relationship between Estrada's downside beta of the individual security and the downside beta of the overall portfolio.

The algorithm presented in this paper sets the value of an investment by searching for the value that generates a bivariate rate of return distribution, such that the investment's marginal contribution to the market portfolio's RTR is infinitely small. The basic assumption is that the cash flow (size) of the individual stock is very small relatively to the cash flow (size) of the whole market. Thus, the weight of each stock in the market is infinitely close to zero but the relative value of individual stocks can be very different since their cash flows are very different. Under equilibrium only the marginal return to risk of all securities should be equal empirically; even the largest firms such as Exxon or Apple are less than 0.0005 of the global equity market. Note that when asymmetrical information is assumed the close to zero optimal proportion of an asset is discarded. When for example there are less-informed investors and a betterinformed investor (e.g., the entrepreneur). The algorithm can be used to calculate the percentage share of an investment that the well-informed entrepreneur would like to retain when 
the less-informed market investors undervalue the future cash flow.

The paper examines the newly proposed algorithm only by highly simplified numerical examples that employed discrete distribution function with few points of return. Thus, the examples with $\mathrm{VaR}$ and AVaR risk measures suffer from discontinuity and non convexity problems. These problems are likely to be minimized in an empirical study that will be based on many more observations. The proposed algorithm can yield empirical "downside betas" and discounting rates for individual securities when various downside risk measures.

The paper examines also the potential impact of idiosyncratic Mean-Variance Preserving Shifts (MVPS) on valuation. Asymmetric information and downside risk considerations drove the effect of such shifts on the entrepreneur's investment retention decisions. The value of the proposed algorithm should be examined by empirical studies that apply the proposed algorithm.

\section{Appendix: Relevant Stochastic Dominance (SD) Principles with a New Theorem}

Rothschild and Stiglitz (1970) defined increasing risk by adding "Mean Preserving Spread" (MPS), which is an atomized unit of additional risk. Let $f(r)$ and $g(r)$ be two alternative distribution functions of a random return $r$, and $F(r)$ and $G(r)$ are their respective cumulative distributions function. Rothschild and Stiglitz proved that the following three claims are equivalent:

1. All risk averters prefer $F$ over $G$.

2. The following integral (SD) condition holds:

$$
\int_{-\infty}^{r}[G(t)-F(t)] d t \geq 0
$$

for every $r$ in the whole range of $r$.

1. The distribution $g$ can be obtained from $f$ by adding a series of MPS steps, where each step is defined by:

$$
\operatorname{MPS}(r) \equiv S(r)=\left\{\begin{array}{l}
+\alpha \quad \text { for } r_{1} \leq r \leq r_{1}+t \\
-\alpha \quad \text { for } r_{1}+a \leq r \leq r_{1}+a+t \\
-\beta \quad \text { for } r_{2} \leq r \leq r_{1}+t \\
+\beta \quad \text { for } r_{2}+b \leq r \leq r_{2}+b+t \\
0 \quad \text { otherwise }
\end{array}\right.
$$

Where:

$$
r_{1} \leq r_{1}+t \leq r_{1}+a \leq r_{1}+a+t \leq r_{2} \leq r_{2}+t \leq r_{2}+b \leq r_{2}+b+t
$$

and:

$$
\alpha a=\beta b 20
$$

We can determine any location of $r_{1}$ and $r_{2}$ provided that $r_{1} \leq r_{2}$, and therefore we can simplify the condition in (A-2) by requiring only $r_{1} \leq r_{2}$ and assuming $\alpha=\beta$ and $a=b$ to obtain:

$$
\operatorname{MPS}(r) \equiv S(r)=\left\{\begin{aligned}
+\alpha & \text { for } r_{1} \leq r \leq r_{1}+t \\
-\alpha & \text { for } r_{1}+a \leq r \leq r_{1}+a+t \\
-\alpha & \text { for } r_{2} \leq r \leq r_{2}+t \\
+\alpha & \text { for } r_{2}+a \leq r \leq r_{2}+a+t \\
0 & \text { otherwise }
\end{aligned}\right.
$$

Where $r_{1} \leq r_{2}, a \geq 0$ and $t \geq 0$.

Figure (A-1) presents the difference between the two distribution functions $g_{i}-f_{i}$; the cumulative distributions functions (CDF), $G_{i}-F_{i}$; and the integral $\int_{-\infty}^{r}\left[G_{i}(t)-F_{i}(t)\right] d t$ due to a single MPS step, as defined in (A-3).

It should be noted that, in Figure 1 , when $r_{2}>r_{l}$, then $F_{i}$ dominates $G_{i}$ according to the Second-Degree Stochastic Dominance (SSD) rule, as $\int_{-\infty}^{r}\left[G_{i}(t)-F_{i}(t)\right] d t \geq 0$ for all $r$. The case where $r_{2}<r_{1}$ is actually an "Anti-Spread". That is, if $G$ is obtained from $F$ by a series of Mean Preserving AntiSpreads (MPAS), then $G$ dominates $F$ by SSD. Adding a spread to $F$ is equivalent to adding an Anti-Spread to $G$.

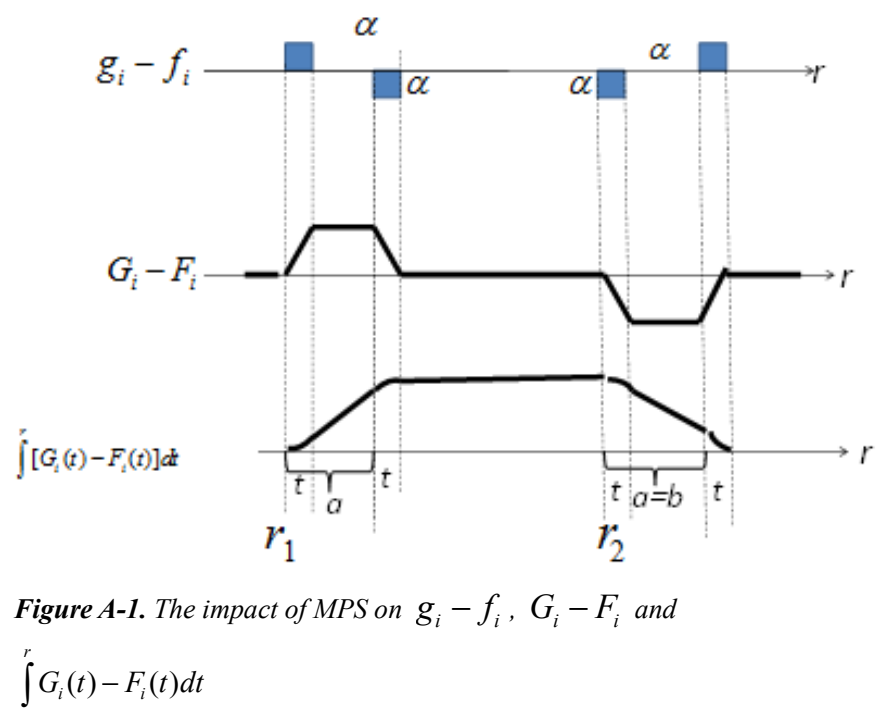

The MPAS can also be defined as follows:

20 This last requirement is necessary and sufficient to obtain equal means for $F$ and $G_{i}$. 


$$
\operatorname{MPAS}(r) \equiv A(r)=\left\{\begin{aligned}
-\alpha & \text { for } r_{3} \leq r \leq r_{3}+t \\
+\alpha & \text { for } r_{3}+c \leq r \leq r_{3}+c+t \\
+\alpha & \text { for } r_{4} \leq r \leq r_{4}+t \\
-\alpha & \text { for } r_{4}+c \leq r \leq r_{4}+c+t \\
0 & \text { otherwise }
\end{aligned}\right.
$$

where $r_{3} \leq r_{4}, c \geq 0$ and $t \geq 0$.

We can also define MPS plus MPAS by:

$$
S A(r) \equiv S(r)+A(r)
$$

Kroll et al. (1995) proved that if $F$ and $G$ have the same means, then $F$ dominates $G$ for all Decreasing Absolute Risk Aversion (DARA) utility investors. This condition holds if and only if there is a sequence $\left\{S A_{i}\right\}_{i=0}^{\infty}$ such that, if added to $f$, the distribution $g$ is generated and in each step $i, S_{i}$ and $A_{i}$ satisfy the TSD rule in (A-6).

$$
\int_{0}^{r} \int_{0}^{v} S_{i}(t) d t d v+\int_{0}^{r} \int_{0}^{v} A_{i}(t) d t d v \geq 0
$$

for all $r$ in the support of the distributions (see Kroll et al. (1995), Theorem 2, pp. 541-542). Kroll et al. (1995) did not provide the necessary and sufficient conditions, under which (12) holds. This rule is provided below in Theorem A-1.

\section{Theorem A-1}

If $a=c$, then $A_{i}(r)$ dominates $S_{i}(r)$ by the TSD rule in (A-6), if and only if $r_{3} \geq r_{1}$ and $r_{4}-r_{3} \leq r_{2}-r_{1}$, where $a, r_{1}$ and $r_{2}$ are defined in (A-3) and $c, r_{3}$ and $r_{4}$ are defined in (A-4).

Proof:

Figure 2-A provides a simple proof of Theorem 1. It depicts the case of one MPS that generates SSD of $F_{i}$ over $G_{i}$, which is equivalent to $\int^{r}\left[G_{i}(t)-F_{i}(t)\right] d t \geq 0$ for all $r$. An MPAS generates similar graphs in Figure 1-A, but with an opposite sign. Namely, an MPAS that starts at $r_{3}$ generates a graph where $\int^{r}\left[G_{i}(t)-F_{i}(t)\right] d t \leq 0$. Thus, the integral is less than or equal to zero, and the MPAS generates SSD of $G_{i}$ over $F_{i}$.

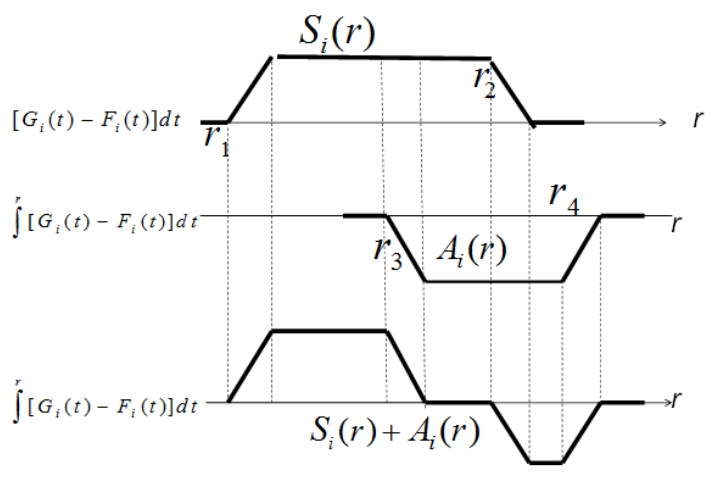

Figure A-2. The impact of $S+A$ on $\int^{r} G_{i}(t)-F_{i}(t) d t$
For $F_{i}$ to dominate $G_{i}$ by Stochastic Dominance (SD) of any degree (including TSD), the left tail of $F_{i}$ must not be above the left tail of $G_{i}$. However, if $r_{3}<r_{1}$, then the left tail of $F_{i}$ is above that of $G_{i}$. Condition (13) for TSD may also be written as:

$$
\int_{0}^{r} \int_{0}^{v} S_{i}(t) d t d v+\int_{0}^{r} \int_{0}^{v} A_{i}(t) d t d v \geq 0 \text { for all } \mathrm{r} .
$$

The double integral over $S_{i}(t)$ for each $r$ is depicted in Figure A-2 by the area between the graph of $\int^{r}\left[G_{i}(t)-F_{i}(t)\right] d t$ and the horizontal axis. Given $t, \alpha$, and $a$, the total area between the graph and horizontal axis is determined by $r_{2}-r_{1}$. A similar graph below the horizontal axis exists if $A_{i}(t)$ is added to $F_{i}(\mathrm{t})$. The total 'negative area' between $\int^{r}\left[G_{i}(t)-F_{i}(t)\right] d t$ and the horizontal axis due to the $A_{i}(\mathrm{t})$, for any given $\mathrm{t}, \alpha$, and $\mathrm{c}$, is determined only by $r_{4}-r_{3}$. Thus if $c=a$ and $r_{4}-r_{3} \leq r_{2}-r_{1}$, then $F_{i}$ dominates $G_{i}$ by TSD, and the accumulated area between $S_{i}(r)+A_{i}(r)$ and the horizontal axis is always positive.

\section{Theorem A- 2}

If $r_{4}>r_{2}$, in addition to the conditions in Theorem A- 1, then $F_{i}$ dominates $G_{i}$ by TSD, but not by SSD.

Proof

Figure A-2 provides a simple proof of Theorem A-2. The lower part of Figure A-2 depicts the effect of $A_{i}+S_{i}$ on the integral $\int^{r}\left[G_{i}(t)-F_{i}(t)\right] d t$. The integral is negative at some points above $r_{2}$, if and only if $r_{4}>r_{2}$. Thus, under this condition there is no $S S D$ of $F_{i}$ over $G_{i}$. On the other hand, the area between $\int^{r}\left[G_{i}(t)-F_{i}(t)\right] d t$ for $A_{i}+S_{i}$ and the horizontal axis is always nonnegative, and there is TSD of $F_{i}$ over $G_{i}$.

The numerical example in Section IV shows that under asymmetric information, when we increase skewness and hold the mean, variance and co-skewness constant, the decision of a better-informed investor may be affected toward higher investment in the firm.

In what follows, we define "Mean-Variance Preserving Shifts" (MVPS).

\section{Theorem A- 3 (MVPS)}

If $G_{i}$ is generated from $F_{i}$ by a single $S A_{i}$ step, then the variance of $G_{\mathrm{i}}$ is equal to the variance $F_{i}$, if and only if $r_{4}-r_{3}=$ $r_{2}-r_{1}$.

Proof The MPS step given in (10) increases the variance of $F_{i}$ according to the equation:

$$
\begin{aligned}
\operatorname{Variance}(M P S) & =\alpha t\left[\left(\mu-r_{1}\right)^{2}-\left(\mu-r_{1}-d\right)^{2}-\left(r_{2}-\mu\right)^{2}+\left(r_{2}+d-\mu\right)^{2}\right] \\
& =2 \alpha t d\left(r_{2}-r_{1}\right)
\end{aligned}
$$


where $\alpha$ measures the shifted probability; $t$ measures the shifted return; $d$ measures the span (distance) of the shifts; and $\mu$ is the mean of $F_{i}$ (see Figure A-3 for the definitions of $\alpha, t, d$ and $\mu$ ). Similarly, MPAS reduces variance by:

$$
\Delta \operatorname{Variance}(M P A S)=-2 \alpha t d\left(r_{4}-r_{3}\right)
$$

Thus, the variance is unchanged due to a Mean Variance Preserving Step (MVPS), which is composed of MPS+ MPAS, if and only if $r_{4}-r_{3}=r_{2}-r_{1}$.

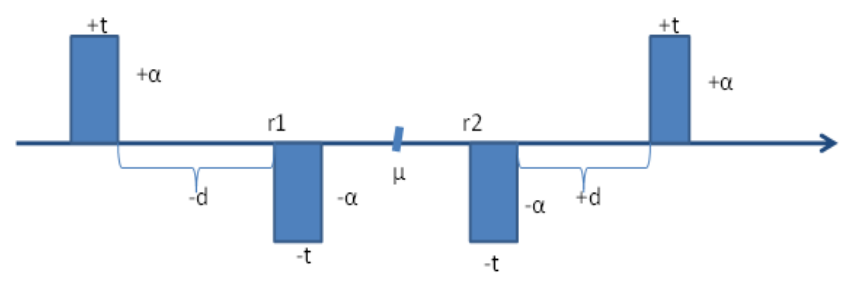

Figures $\boldsymbol{A}$-3. The parameters of MPS

Note that, in the case of MVPS, there is only TSD of $F_{i}$ over $G_{i}$ because the conditions $r_{3}>r_{1}$ and $r_{4}-r_{3}=r_{2}-r_{1}$ imply $r_{4}>r_{2}$, which, according to Theorem 3 , is the condition under which $F_{i}$ dominates $G_{i}$ by TSD, but not by SSD

\section{Theorem A- 4}

$G_{i}$ has a lower skewness $\left(\mu_{3}\right)$ than that of $F_{i}$ if we generate $G_{i}$ by adding a single MVPS to $F_{i}$ and if the anti-spread starts to the right of the spread $\left(r_{1}<r_{3}\right)$.

Proof The change in skewness $\Delta \mu_{3}$ due to a single MPS is given by:

$\Delta \mu_{3}(M P S)=\alpha t\left[\left(r_{1}-\mu\right)^{3}-\left(r_{1}-\mu+d\right)^{3}-\left(r_{2}-\mu\right)^{3}+\left(r_{2}-\mu+d\right)^{3}\right]$ With some algebra, we obtain: $\Delta \mu_{3}(M P S)=-3 \alpha t d\left(r_{2}-r_{1}\right)\left[2 \mu-d+r_{1}+r_{2}\right]$

Note that MPS generates positive skewness only if $d<2 \mu+r_{1}+r_{2}$. Similarly, the effect of MPAS on skewness is: $\Delta \mu_{3}(M P A S)=-3 \alpha t d\left(r_{4}-r_{3}\right)\left[2 \mu-d+r_{3}+r_{4}\right]$

The requirement $r_{4}-r_{3}=r_{2}-r_{1}$ preserves the variance, and therefore the requirement $r_{1}<r_{3}$ is necessary and sufficient for the skewness of $G_{i}$ to be less than that of $F_{i}$.

Note that, MVPS shifts do not changes mean and variance. Thus valuation according to mean- variance criterion does not reflect such changes. The numerical example that is provided in this paper demonstrates that MVPS shifts that are actually idiosyncratic changes of risk, may affect valuation when the valuation is based on down side risk measure and asymmetrical information is assumed.

\section{References}

[1] Ang, A., J. Chen and Y. Xing. 2006. Downside risk. The Review of Financial Studies. 19 1191-1239.

[2] Arditti, F. D. 1967. Risk and the required return on equity. Journal of Finance, 22 19-36.

[3] Baumol, W. J. 1963. An Expected gain-confidence limit criterion for portfolio selection. Management Science, 10174 181 .
[4] Bawa, V.S. and E. B. Lindenberg 1977. Capital market equilibrium in a mean-lower partial moment framework. Journal of Financial Economics. 5 189-200.

[5] Bawa, V. S. 1978. Safety-First, stochastic dominance and optimal portfolio choice. Journal of Financial and Quantitative Analysis. 13 255-271.

[6] Barone A., G. Gagliardiniand, G. Urga 2004. Testing asset pricing models with co-skewness. Journal of Business and economic Statistics. 22 474-485.

[7] Brockett, P. L., and L. L. Golden 1987. A Class of utility functions containing all the common utility functions. Management Science. 33, 8 955-964.

[8] Cheremushkin, S. 2009. Why D-CAPM is a big mistake? The incorrectness of the co-semi-variance statistics. Available at SSRN: http://ssrn.com/abstract 1336169.

[9] Dittmar, R. F. 2002. Non-linear pricing kernels, kurtosis, preferences, and evidence from cross-section of equity returns," Journal of Finance. 57 369-402.

[10] Danielson J., B. J. Jorgensen, M. Sarma, M. and C.J. Vries 2006. Comparing downside risk measures for heavy tailed distributions. Economic Letters. 92 202-208.

[11] Estrada, J. 2007. Mean semi-variance behavior: Downside risk and capital asset pricing. International Review of Economics and Finance. 16 169-185.

[12] Fama, E. F., and K. R. French, 1992. The Cross-Section of Expected Stock Returns, Journal of Finance. 47, 427-465.

[13] Fama, E. F., and K. R. French, 1993. Common Risk Factors in the Returns on Stock and Bonds, Journal of Financial Economics, 33, 3-56.

[14] Fama, E. F., and K. R. French, 1995. Size and Book-to-Market Factors in Earnings and Returns. Journal of Finance, 50, 131155.

[15] Fishburn, P.C. 1977. Mean-Risk analysis with risk associated with below target returns. American Economic Review. 67, 817-836.

[16] Friend, I. and R. Westerfield 1980. Co-Skewness and capital asset pricing. Journal of Finance. 35 897-913.

[17] Hadar, J. and W. R. Russell 1971. Stochastic dominance and diversification, Journal of Economic Theory. 3 288-305.

[18] Hanoch, G., and H. Levy 1969. Efficiency analysis of choices involving risk. Review of Economic Studies. 36 335-346.

[19] Harvey, C. R. and A. Siddique 2000. Conditional skewness in asset pricing tests. Journal of Finance 55 1263-1295.

[20] Javid A. Y. 2009. Test of higher moment capital asset pricing model in case of pakistani equity market." European Journal of Economics, Financial and Administrative Sciences.15 144162.

[21] Kraus, A. and R. Litzenberger 1976. Skewness Preference and the Valuation of Risky Assets", Journal of Finance. 31 10851094.

[22] Kroll, Y., M. Leshno, H. Levy and Y. Spector 1995. Increasing risk, decreasing absolute risk aversion and diversification. Journal of Mathematical Economics.24 537-556. 
[23] Levy, H. and H. M. Markowitz 1979. Approximating expected utility by a function of mean and variance. American Economic Review. 69 308-317.

[24] Magni, C.A. 2007. Project valuation and investment decisions: CAPM versus arbitrage. Applied Financial Economics Letters. 3 137-140.

[25] Magni, C.A. 2009. Correct or incorrect application of the CAPM? European Journal of Operational Research, 192 549560.
[26] Markowitz, H. 1990. Foundation of portfolio theory: Nobel Lecture". Economic Sciences, 279-287.

[27] Nawrocki, D. 1999. A brief history of downside risk measures. The Journal of Investing, 8 9-25.

[28] Post T., and P.V. Vilet 2006. Downside risk and asset pricing. Journal of Banking and Finance. 30 823-840.

[29] Rothschild M. and J. E. Stiglitz. 1970. Increasing risk: I. A definition. Journal of Economic Theory, 2 225-243. 